EPJ Web of Conferences 61, 08007 (2013)

DOI: $10.1051 /$ epjconf/ 20136108007

(C) Owned by the authors, published by EDP Sciences, 2013

\title{
VERA monitoring of a blazar OJ 287
}

\author{
S. Sawada-Satoh ${ }^{1, a}$, K. Akiyama ${ }^{2,4}$, M. Kino ${ }^{3}$, H. Nagai ${ }^{4}$, K. Niinuma ${ }^{5}$, and The GENJI Team \\ ${ }^{1}$ Mizusawa VLBI Observatory/NAOJ, Hoshigaoka-cho, Mizusawa-ku, Oshu, Iwate, 023-0861 Japan \\ ${ }^{2}$ The University of Tokyo, Hongo, Bunkyo-ku, Tokyo, 113-8654 Japan \\ ${ }^{3}$ ISAS/JAXA, Yoshinodai, Chuo-ku, Sagamihara, Kanagawa, 252-5210 Japan \\ ${ }^{4}$ NAOJ, Osawa, Mitaka, Tokyo, 181-8588 Japan \\ ${ }^{5}$ Yamaguchi University, Yoshida, Yamaguchi, Yamaguchi 753-8512
}

\begin{abstract}
We present the frequent VLBI monitoring results of blazar OJ 287 at $22 \mathrm{GHz}$ band using the VLBI Exploration of Radio Astrometry (VERA) from 2010 November to 2012 September. Time interval of the monitoring is typically once or twice per month. The 22-GHz light curve of OJ 287 show three obvious increasing activities around 2011 May, 2011 October, and 2012 March, with a timescale of $<4$ months. The second radio increasing events occurred at the same time as the $\gamma$-ray flare in 2011 October detected by the Fermi Large Area Telescope (LAT), and the third one seemed to precede the $\gamma$-ray flares in 2012, exhibiting different behavior from the previous $\gamma$-ray flaring events in 2009. The relative motion of one jet component from the core was rectilinear super luminal motion $(\sim 8 c)$ from the 2011 February to 2011 November toward the south direction, and then it changed the direction backward. The direction variation of the relative motion was seen from 2011 November to 2012 August, simultaneously with the $\gamma$-ray flares. It could be caused by a new ejected component in the innermost region.
\end{abstract}

\section{Introduction}

OJ 287 is one of the most observed blazar at a redshift of 0.306[1], and exhibits periodic double-peaked optical outburst at a period of $\sim 12$ years[2][3][4]. Besides the double-peaked outburst every $\sim 12$ year, this source is known to show a rapid variability across a wide range of wavelength. Recently, an increasing activity at highenergy band like $\gamma$-ray and X-ray was detected by Fermi LAT[5] and Swift X-Ray Telescope[7] in 2011 October, and this flare was also seen at infra-red and optical wavelengths. And in 2012 March, another increasing event was detected at optical and X-ray wavelengths [6][7]. After that, a $\gamma$-ray detection by Fermi LAT was reported at a level of $1 \times 10^{-6}$ photons $\mathrm{cm}^{-2} \mathrm{~s}^{-1}$ from 2012 May to June[8][9].

Very Long Baseline Interferometry (VLBI) monitoring toward blazars allows us to identify the location of the flaring event in parsec scale. The time scale of the flux variability seen in blazars is often shorter than one month[10][11], and therefore it is important to carry out frequent VLBI monitoring observations at shorter time interval than one month. Here, we report on the results of VERA monitoring of OJ 287 for 2 years from 2010 to 2012. This research is a part of the monitoring observations GENJI (Gamma-ray Emitting Notable AGN Monitoring by Japanese VLBI[12]), which is a monitoring program of $\gamma$-ray bright AGNs with the VERA.

\footnotetext{
ae-mail: satoko.ss@nao.ac.jp
}

\section{Observations and data reductions}

VLBI observations were carried out toward OJ 287 from 2010 November to 2012 September at $22 \mathrm{GHz}$ with the VERA, which consists of four 20-m antennas. The onsource time for each observation of OJ 287 was typically 30 minutes, spreading into several scans of 5 minutes, because OJ 287 was observed as a calibrator source for the VERA astrometric observations.

Data reduction including calibration, data flagging, fringe fitting, and imaging self calibration was done using the NRAO AIPS package. A priori amplitude calibration was performed using the AIPS task APCAL based on measurements of the system temperature during observations and the antenna gain information for each VERA station. Uncertainties in the amplitude calibration are $10 \%$ or less at $22 \mathrm{GHz}$ [13]. For some observations, we combined multiple visibility datasets taken within a few days so as to improve imaging qualities. Time interval of the monitoring is once or twice per month.

After the imaging, we fitted three Gaussians (the core: $\mathrm{C} 1$, the jet component: $\mathrm{C} 2$, the inner jet component located between $\mathrm{C} 1$ and $\mathrm{C} 2$ : $\mathrm{C} 3$ ) to the images using the AIPS task JMFIT, in order to estimate the position and flux density of each component. The relative positional accuracy of a component in the Gaussian fitting was within 0.02 mas, depending on the signal-to-noise ratio. We adopt $H_{0}=71 \mathrm{~km} \mathrm{~s}^{-1} \mathrm{Mpc}^{-1}, \Omega_{\mathrm{M}}=0.27$ and $\Omega_{\Lambda}=0.73$, and hence 1 mas corresponds to $4.5 \mathrm{pc}$, and $1 \mathrm{mas} \mathrm{yr}^{-1}$ corresponds to $19 c$ at the distance of OJ 287. 


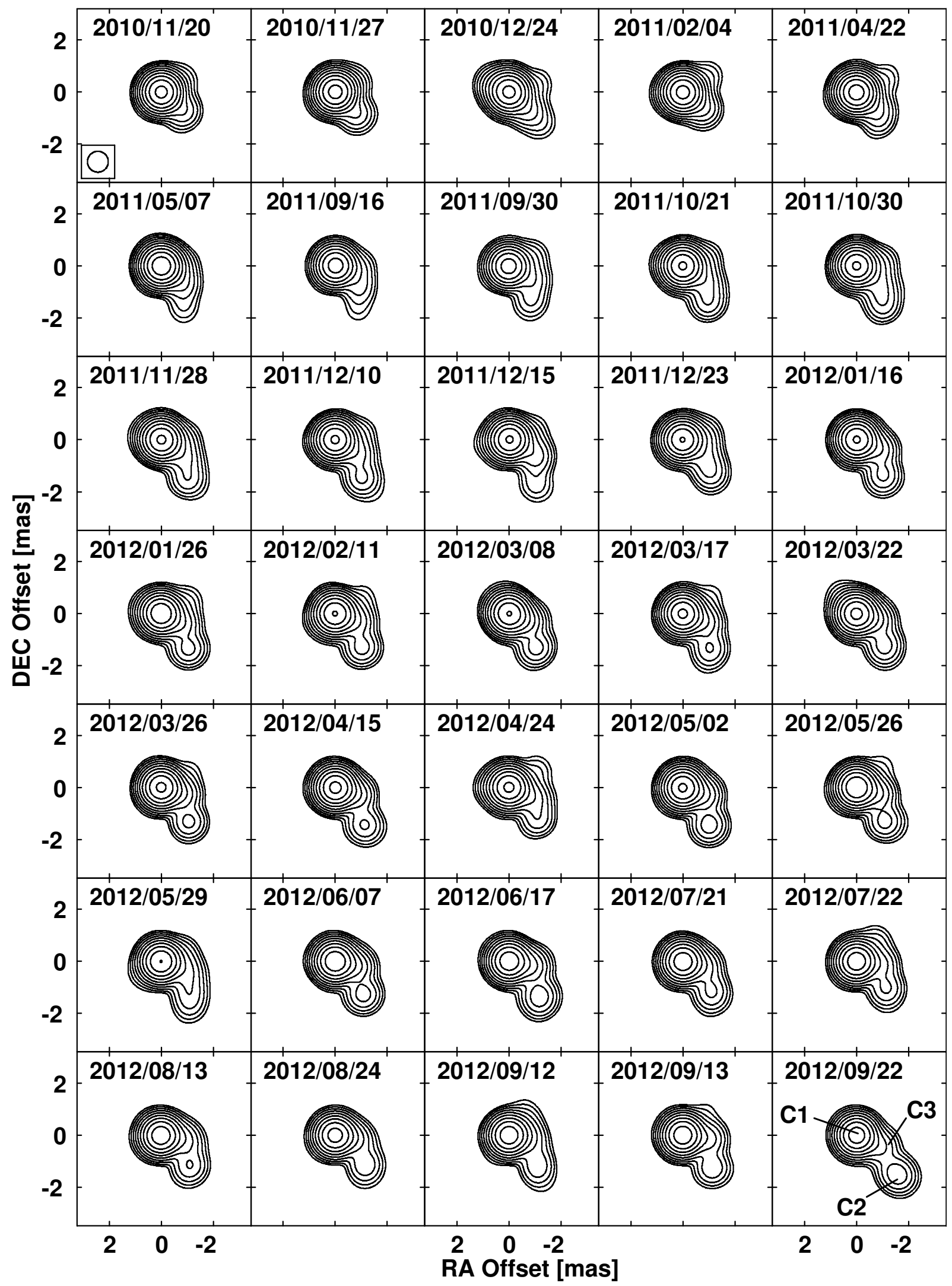

Figure 1. Sequence of VERA 22-GHz images of OJ287 from 2010 November to 2012 September. Images are convolved with a circular beam size (FWHM) of 0.8 mas, which corresponds to the typical minor axis of synthesized beam in our observations. Contours start at $9 \mathrm{mJy}_{\text {beam }}{ }^{-1}(3 \sigma$ level$)$, increasing by a factor of 2 . The structures can be resolved into three components, the core $(\mathrm{C} 1)$, the jet component (C2) and the inner jet component located between $\mathrm{C} 1$ and $\mathrm{C} 2(\mathrm{C} 3)$. 


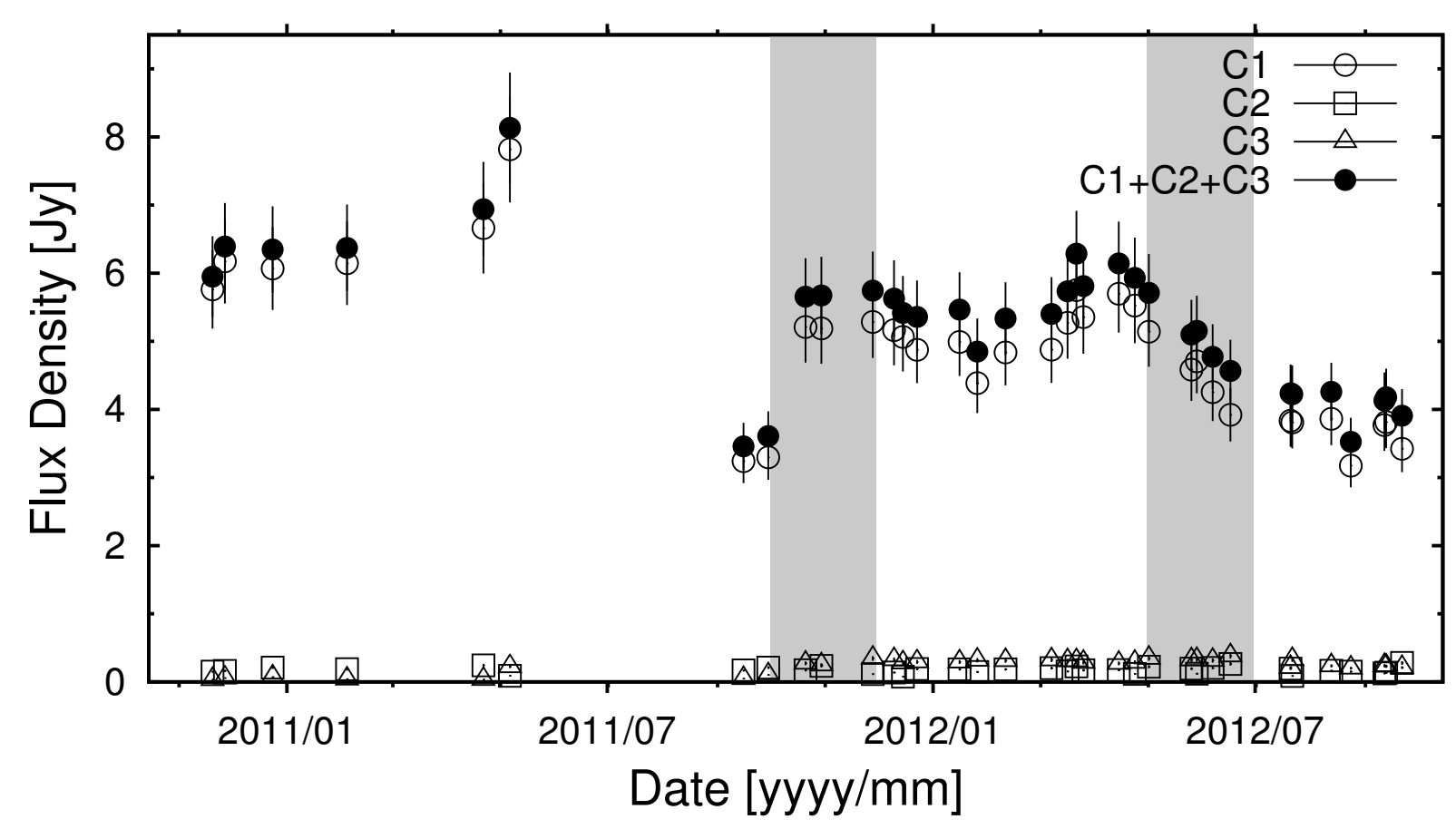

Figure 2. Light curve of each component in OJ 287 at $22 \mathrm{GHz}$. We adopted $10 \%$ for the flux error. Two $\gamma$-ray flares at a level of $1 \times 10^{-6}$ photons $\mathrm{cm}^{-2} \mathrm{~s}^{-1}$ are indicated by gray rectangles.

\section{Results}

\subsection{Parsec-scale structure}

Figure 1 shows the pc-scale core-jet structure, which is well represented by three components; the core $(\mathrm{C} 1)$, the jet component (C2) and the inner jet component (C3). The components $\mathrm{C} 1$ and $\mathrm{C} 2$ could be identified as ' $\mathrm{C}$ ' and ' $\mathrm{j}$ ' of the VLBA 43-GHz images[14]. Then, the innermost jet components 'a', 'm', 'n', 'o' and 'p' could be included into the components $\mathrm{C} 1$ or $\mathrm{C} 3$, because the angular resolution of our images is 5 times lower than that of the VLBA 43$\mathrm{GHz}$ images.

\subsection{Radio light curve}

The 22-GHz light curve of the component $\mathrm{C} 1$ in OJ 287 by GENJI/VERA reveals three increasing activities in 2011 and 2012. The first one occurred between 2011 May and September at the peak flux density of $\geq 7.8 \mathrm{Jy}$. The 22$\mathrm{GHz}$ flux density reached the minimum in 2011 September, and increased again. This is the second increasing activity. And the third one started to rise from 2012 January and reached the peak in 2012 March with a flux density of $\sim 6$ Jy (figure 2). The time scale of those increasing activities can be estimated to be $<4$ months as a period between the onsets of rise and decay in flux.

In this period, two pronounced $\gamma$-ray flares in OJ 287 are detected by Fermi LAT, in 2011 October and 2012 May. The two $\gamma$-ray flaring events seem to occur with/after the radio increasing events, unlike the previous $\gamma$-ray flaring events in 2009[15].

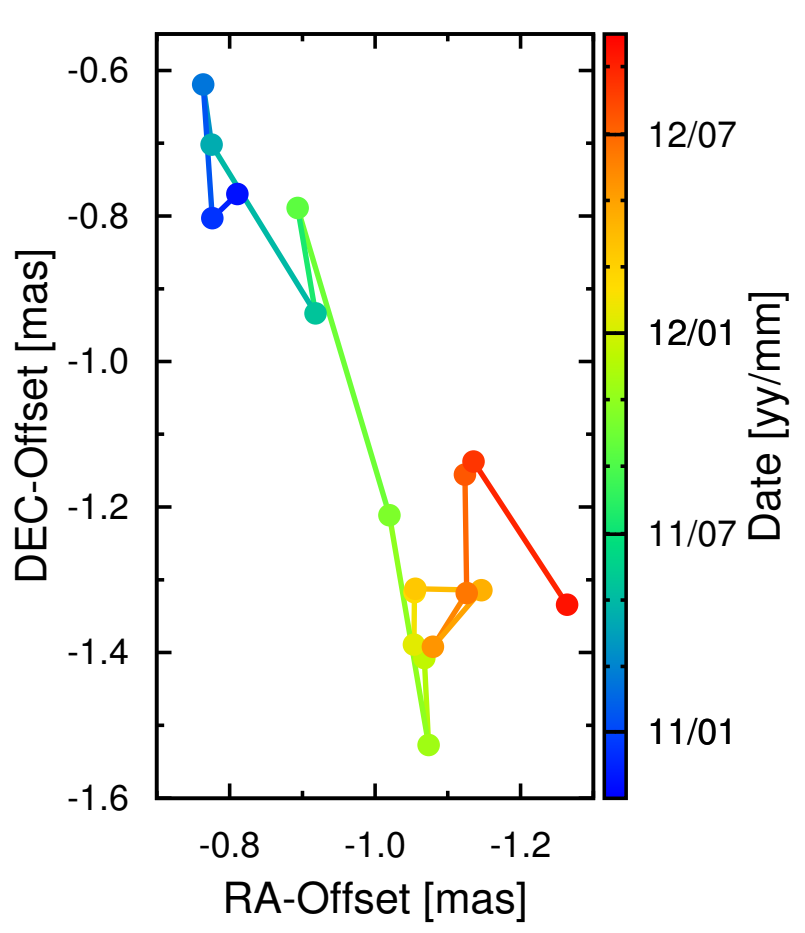

Figure 3. Relative motion of the component $\mathrm{C} 2$ with respect to the component $\mathrm{C} 1$. Data points are obtained averaging by month from 2010 November to 2012 September, expect January, March, June, July and August in 2011. The standard deviation of the monthly average is typically $\sim 0.1$ mas.

\subsection{Jet motion}

Figure 3 indicates the relative motion of the component $\mathrm{C} 2$ with respect to the component $\mathrm{C}$ 1. Overall, the angular 
separation between the components $\mathrm{C} 1$ and $\mathrm{C} 2$ increased $\sim 0.8$ mas from 2010 November to 2012 September. It corresponds to a superluminal motion with an apparent velocity of $\sim 8 c$, applying rectilinear motion. The superluminal speed is consistent with the velocity $(6.7 \pm 0.4 c)$ of the jet component ' $\mathrm{j}$ ' measured with the VLBA at $43 \mathrm{GHz}$ [14].

From 2010 November to 2011 April, the relative position was stalled to the position at $(-0.8,-0.7)$ in figure 3 , and then moved along the direction at the position angle of $\sim-160^{\circ}$ until 2011 November. After that, the motion of the component $\mathrm{C} 2$ rapidly changed the direction to backward from 2011 November to 2012 August. The time scale of the jet-direction variation is shorter than one year. This jet-direction variation was seen in the high state period of $\gamma$-ray with two flares in 2011 and 2012. Such a synchronization between the jet position angle change and the core flux brightening has been reported in the innermost jet region mapped with the VLBA $43 \mathrm{GHz}$ monitoring[14].

\section{Discussion}

The relative motion between the component $\mathrm{C} 1$ and $\mathrm{C} 2$ should be affected by both positions of the components $\mathrm{C} 1$ and $\mathrm{C} 2$, and thus the direction variation of the relative position could be due to wobbling behavior of either or both of $\mathrm{C} 1$ and $\mathrm{C} 2$. The component $\mathrm{C} 1$ could include contribution from the innermost jet components. No newborn jet component was seen in our VLBI images at the period the jet direction varied. However, the relative position of the component C2 from 2011 November to 2012 August moved backward and forward along a line at a position angle of $\sim-20^{\circ}$, nearly parallel to the ejection direction of the inner jet component 'a' detected by the VLBA $43 \mathrm{GHz}$ monitoring[14]. This fact supports the core wobbling hypothesis, which means that the direction variation could be caused by the ejection of a new component, which is not be resolved with our observations. In order to confirm our result, measurements of the absolute proper motions of the components with the phase referencing VLBI observations are necessary.

\section{Acknowledgment}

We are grateful to all staff members of VERA for their efforts and continuous support on the observations. This work is partially supported by a Grand-in-Aid for Science Research, KAKENHI 24540240 (MK) from Japan Society for the Promotion of Science (JSPS).

\section{References}

[1] Stickel M., Fried J. W., Kuehr H. A\&AS, 80, 103 (1989)

[2] Sillanpaa, A., Haarala, S., Valtonen, M. J., Sundelius, B., \& Byrd, G. G. ApJ, 325, 628 (1988)

[3] Sillanpaa, A., Takalo, L. O., Pursimo, T., et al. A\&A, 315, L13 (1996)

[4] Lehto, H. J., \& Valtonen, M. J. ApJ, 460, 207 (1996)

[5] Escande, L., \& Schinzel, F. K. The Astronomer's Telegram, 3680 (2011)

[6] Pihajoki, P., Valtonen, M., Zola, S., et al. ApJ, 764, 5 (2013)

[7] Stroh, M. C., \& Falcone, A. D. ApJS, 207, 28 (2013)

[8] http://fermisky.blogspot.jp/2012/05/fermi-lat-weeklyreport-n-206.html

[9] http://fermisky.blogspot.jp/2012/06/fermi-lat-weeklyreport-n.html

[10] Lazio, T. J. W., Waltman, E. B., Ghigo, F. D., et al. ApJS, 136, 265 (2001)

[11] Kadota, A., Fujisawa, K., Sawada-Satoh, S., Wajima, K., \& Doi, A. PASJ, 64, 109 (2012)

[12] Nagai, H., Kino, M., Niinuma, K., et al. PASJ, 65, 24 (2013)

[13] Petrov, L., Honma, M., \& Shibata, K. M. AJ, 143, 35 (2012)

[14] Agudo, I., Marscher, A. P., Jorstad, S. G., et al., ApJ, 747, 63 (2012)

[15] Agudo, I., Jorstad, S. G., Marscher, A. P., et al. ApJL, 726, L13 (2011) 